\title{
Mono- and bis-alkylation of glyoxal-bridged tetraazamacrocycles using mechanochemistry
}

Bassim H. Abdulwahaab, Benjamin P. Burke, Juozas Domarkas, Jon D. Silversides, Timothy J. Prior and Stephen J. Archibald ${ }^{\dagger *}$

Electronic Supplementary Information

NMR

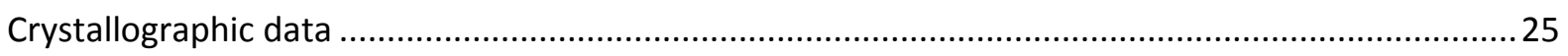


NMR

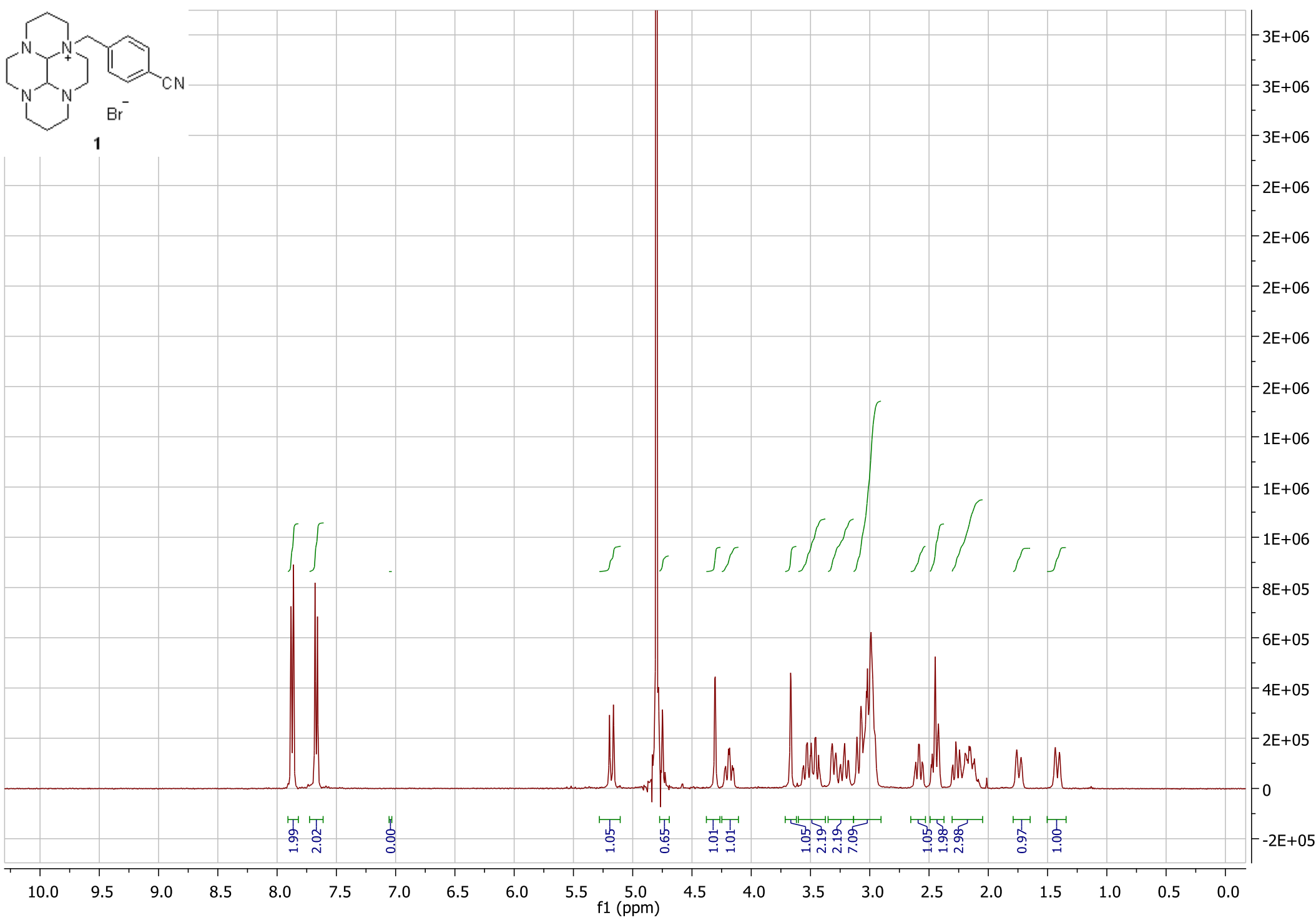




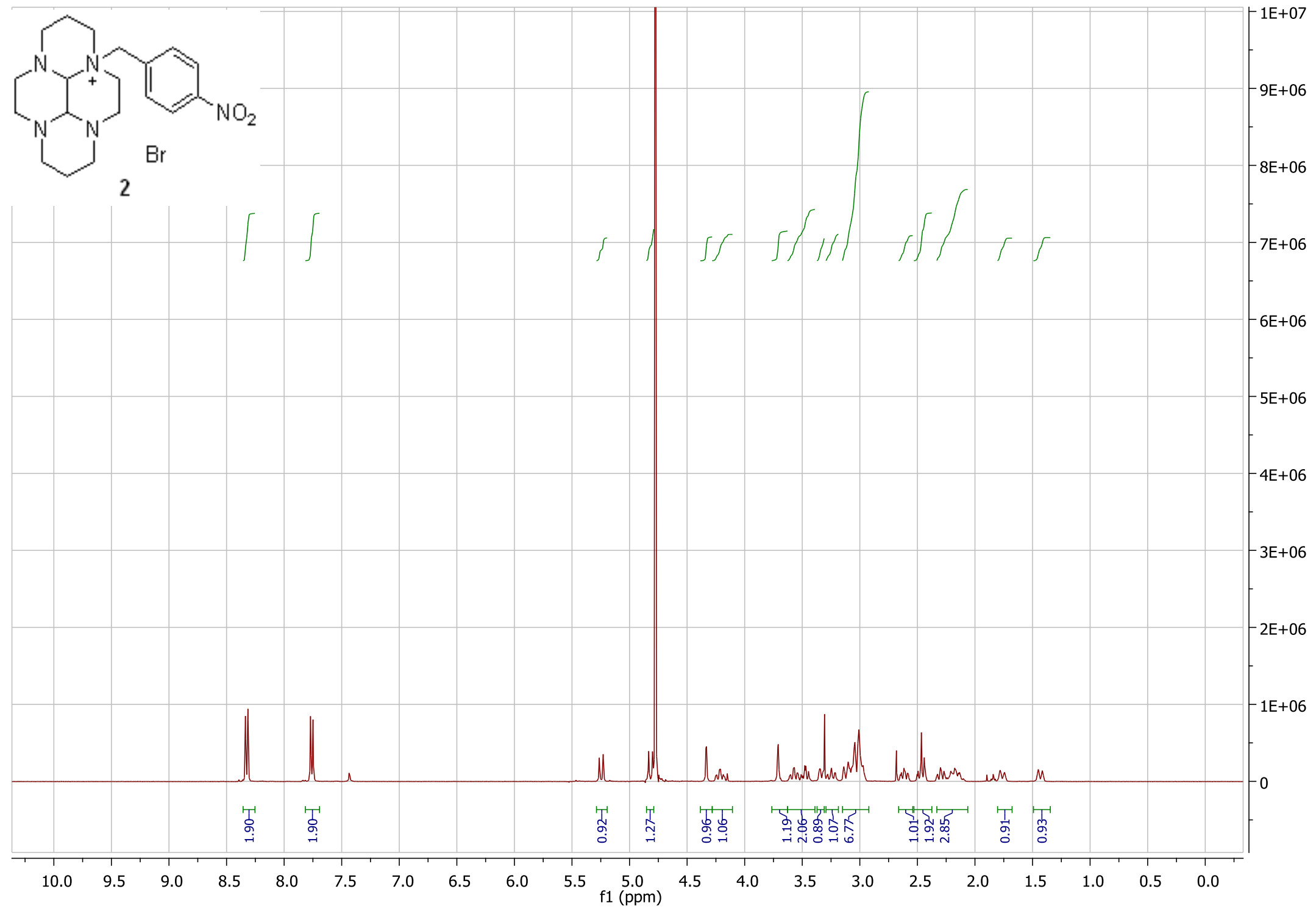




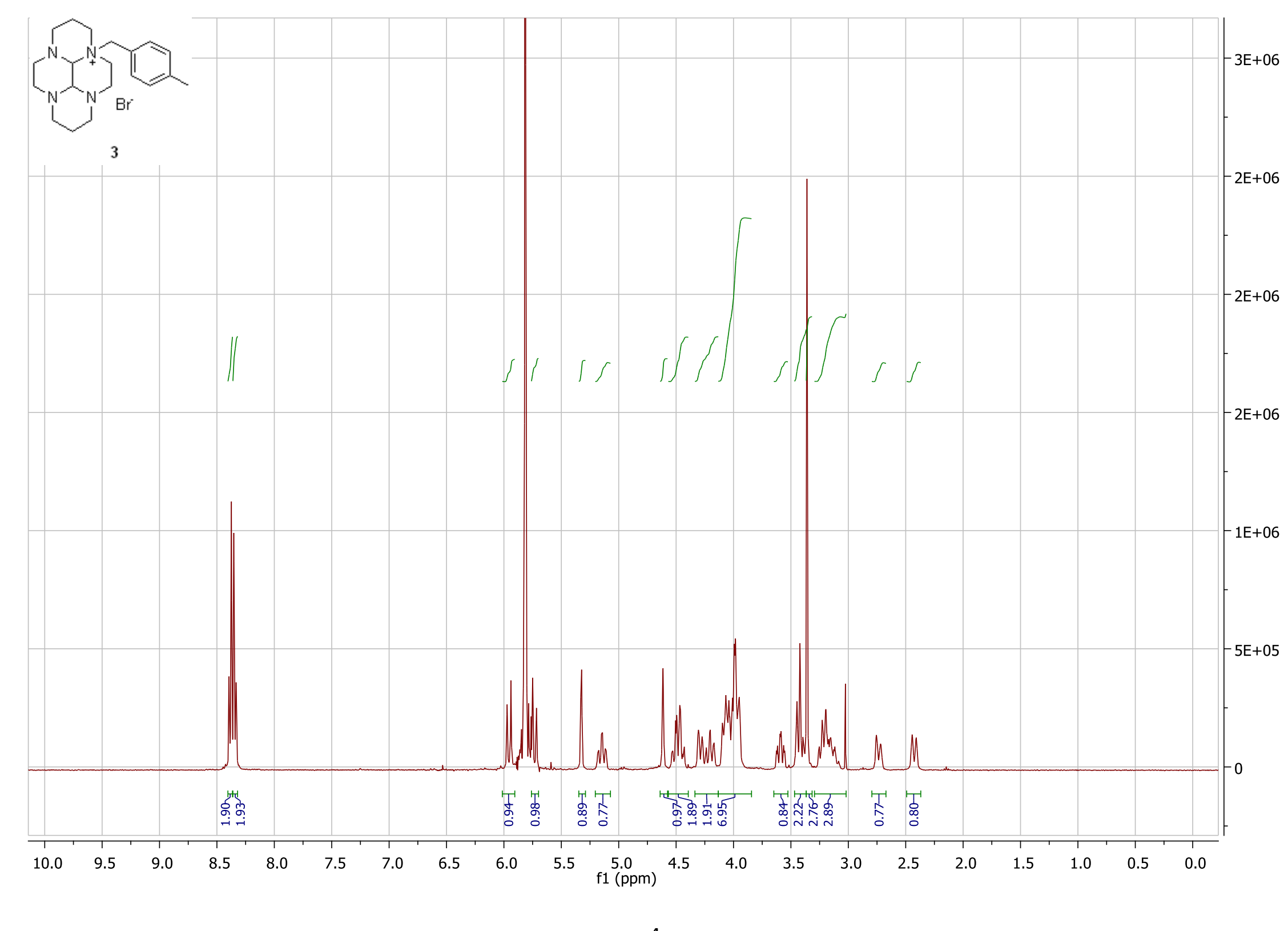




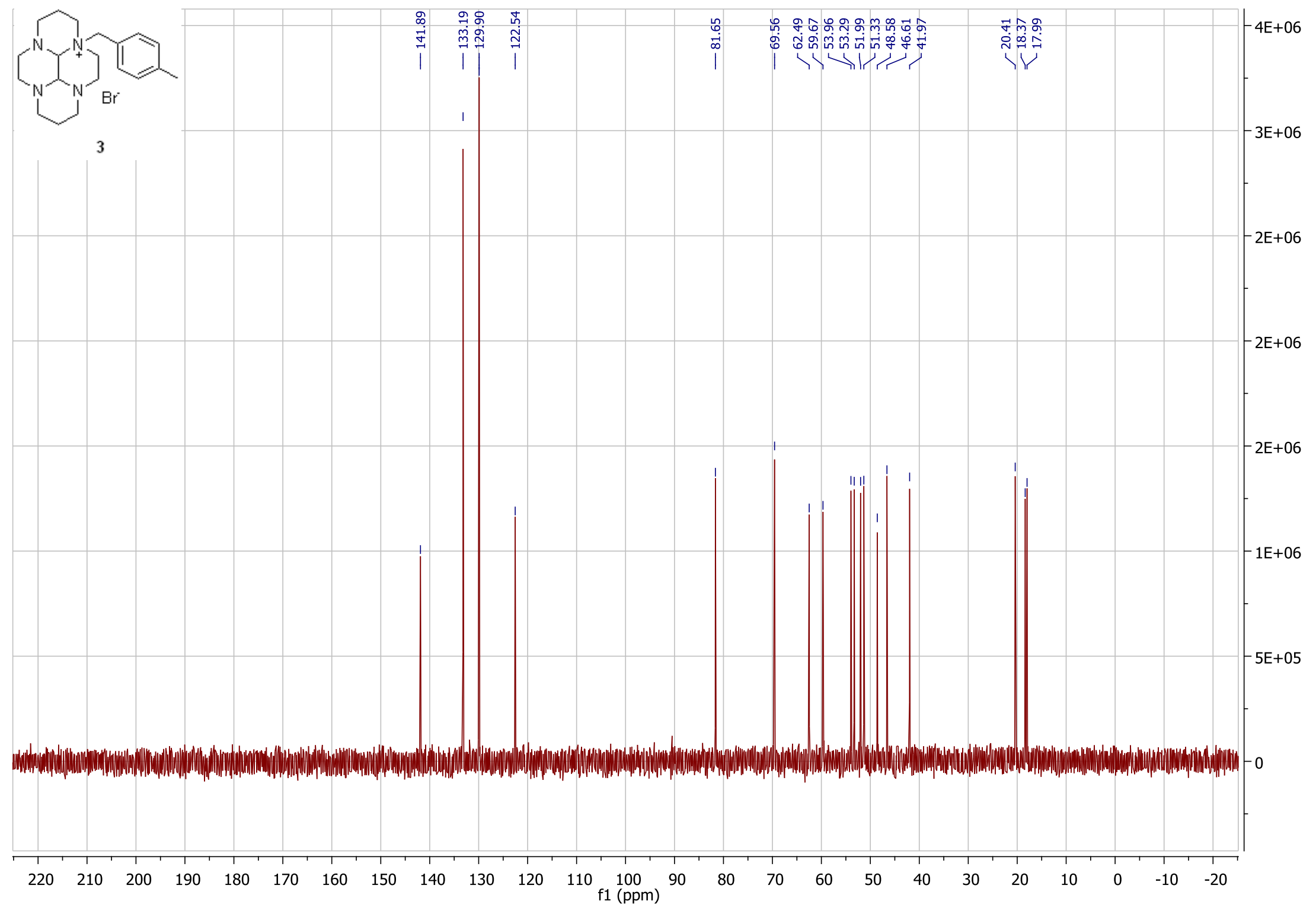




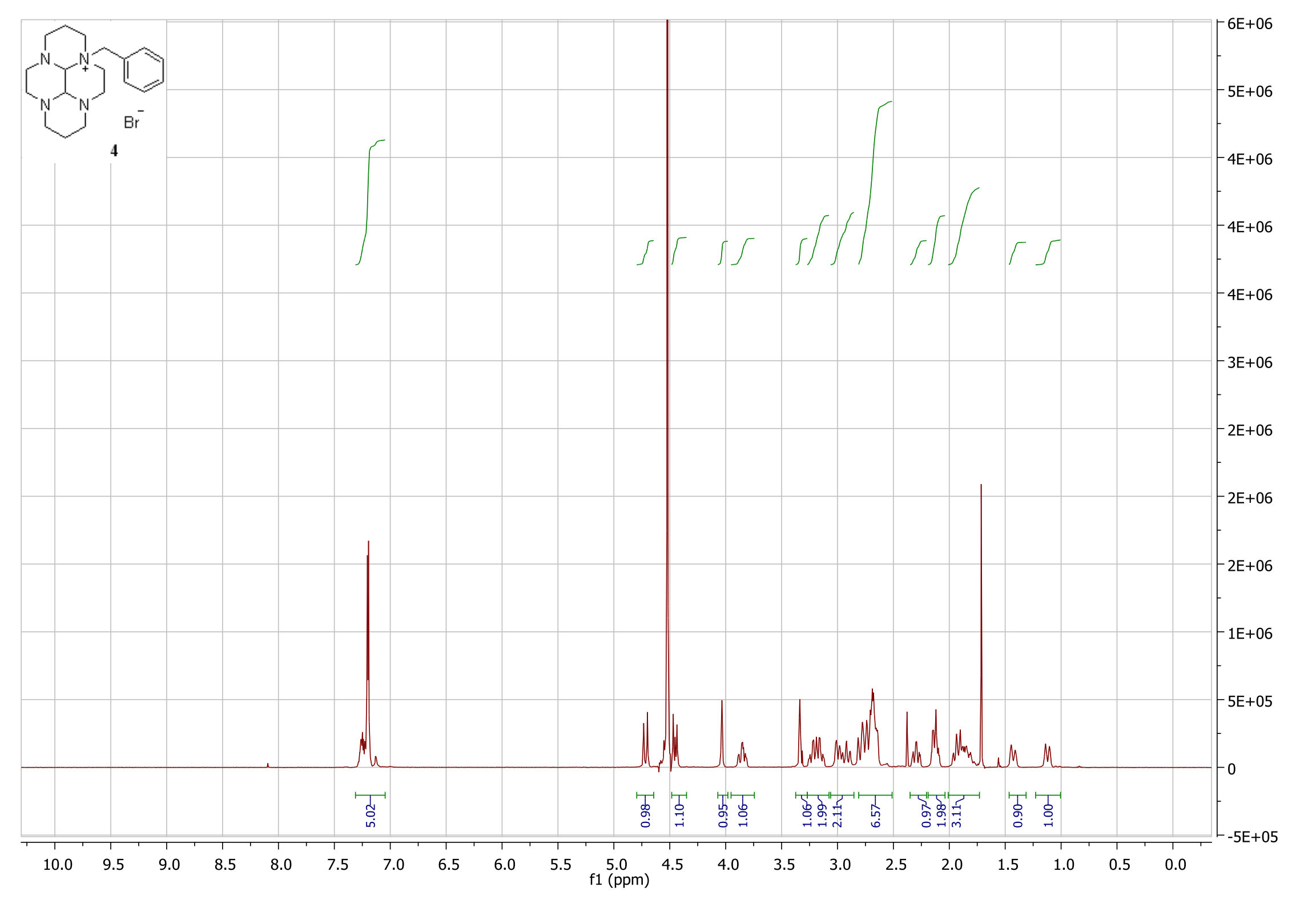




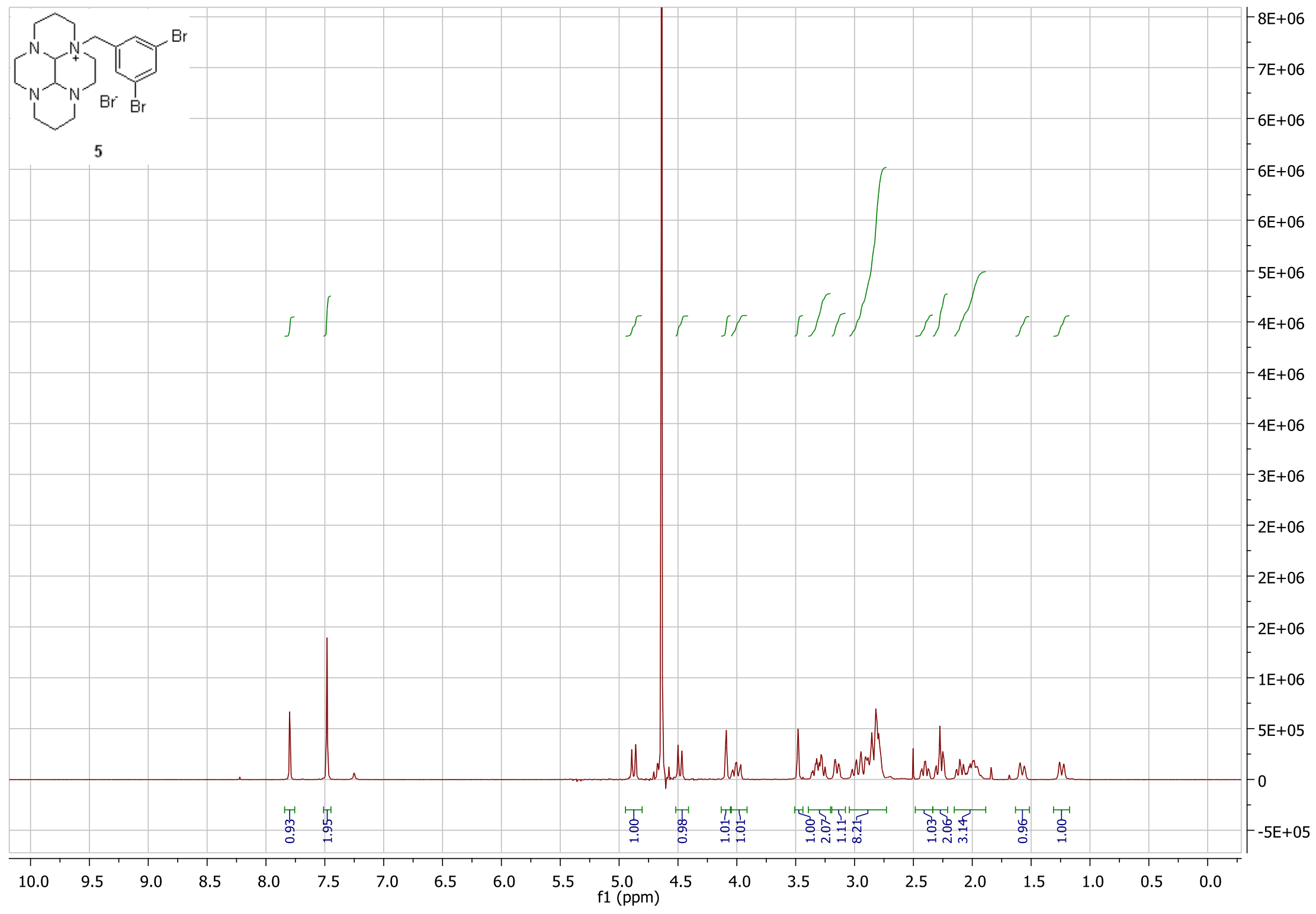




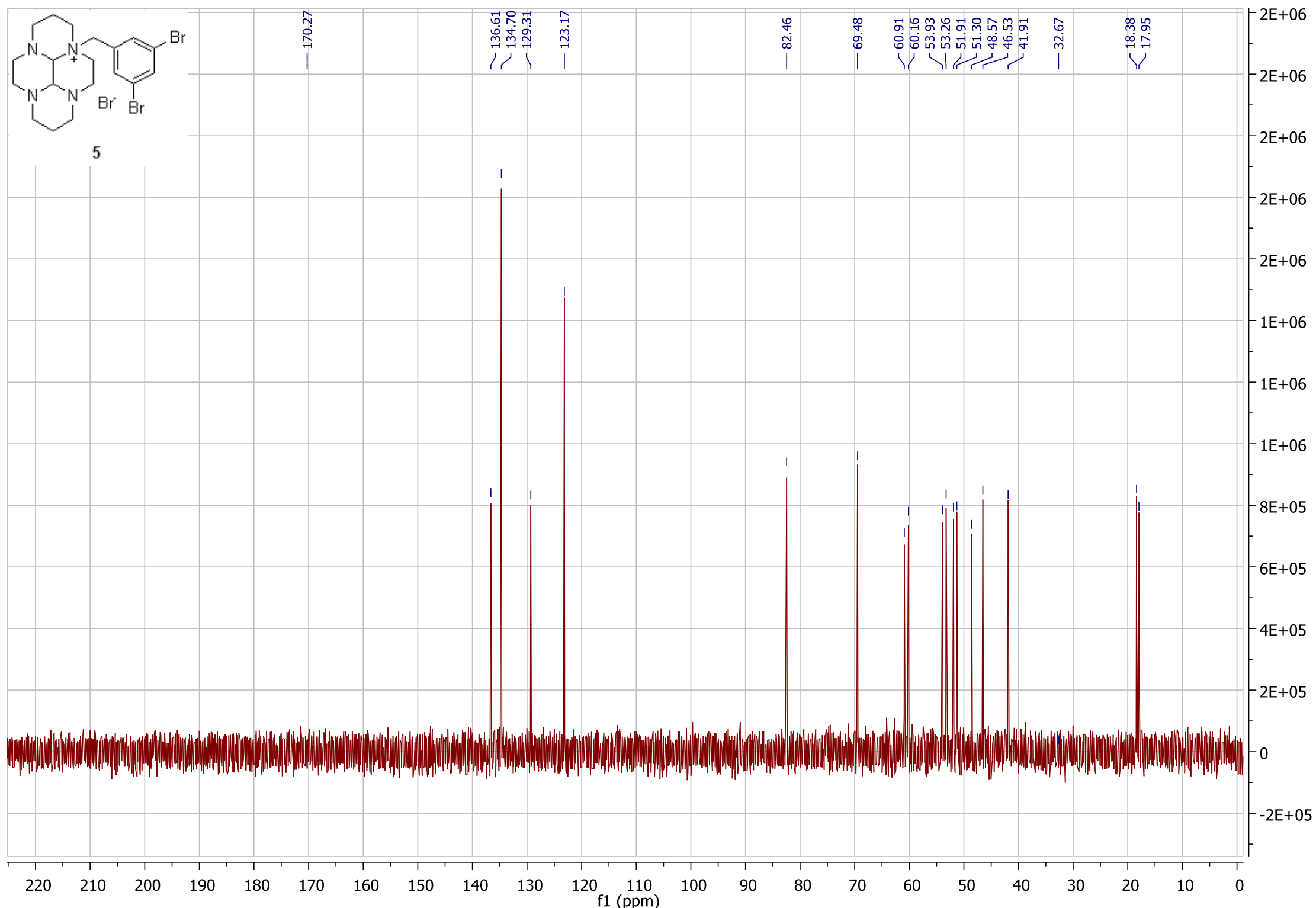




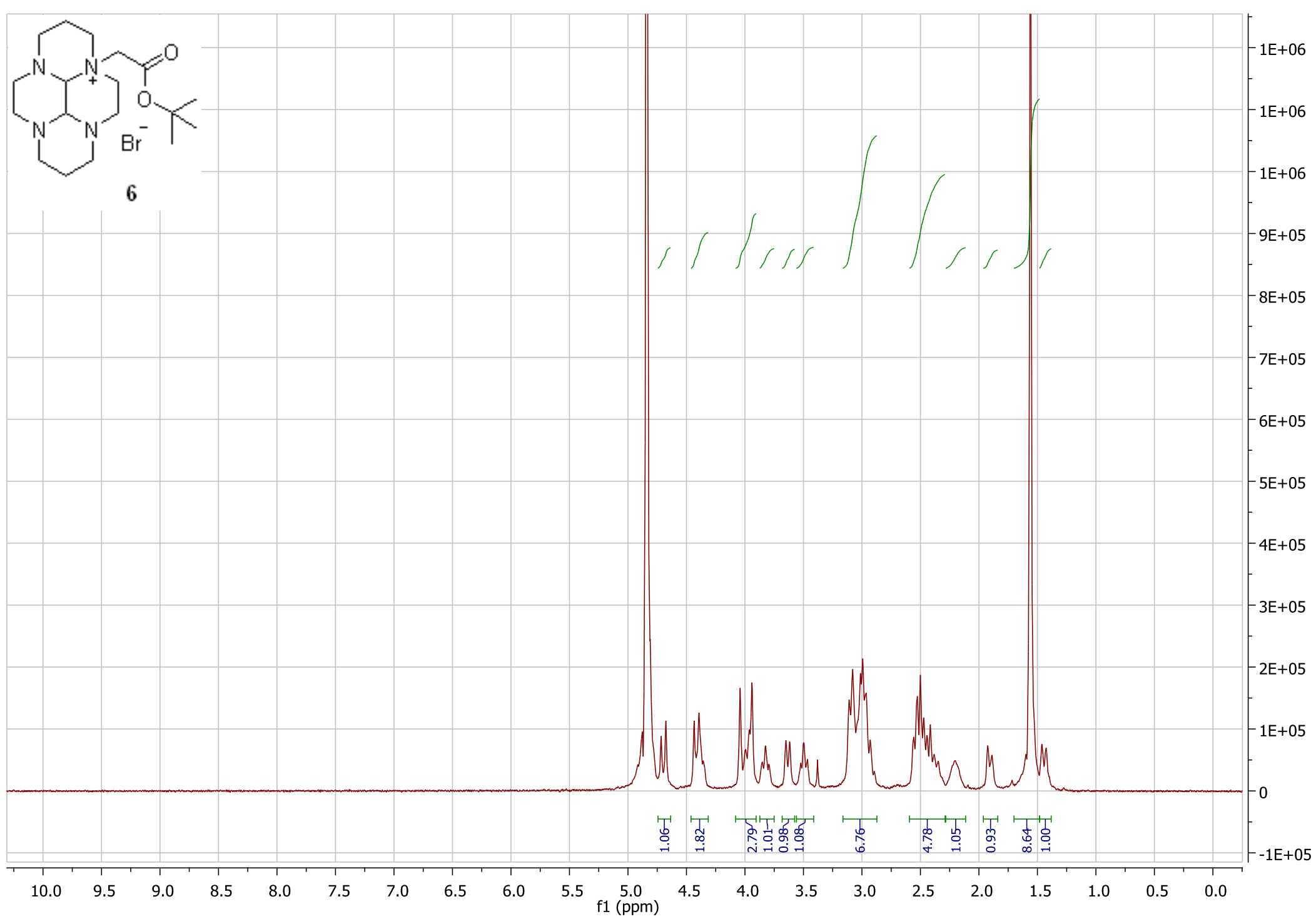




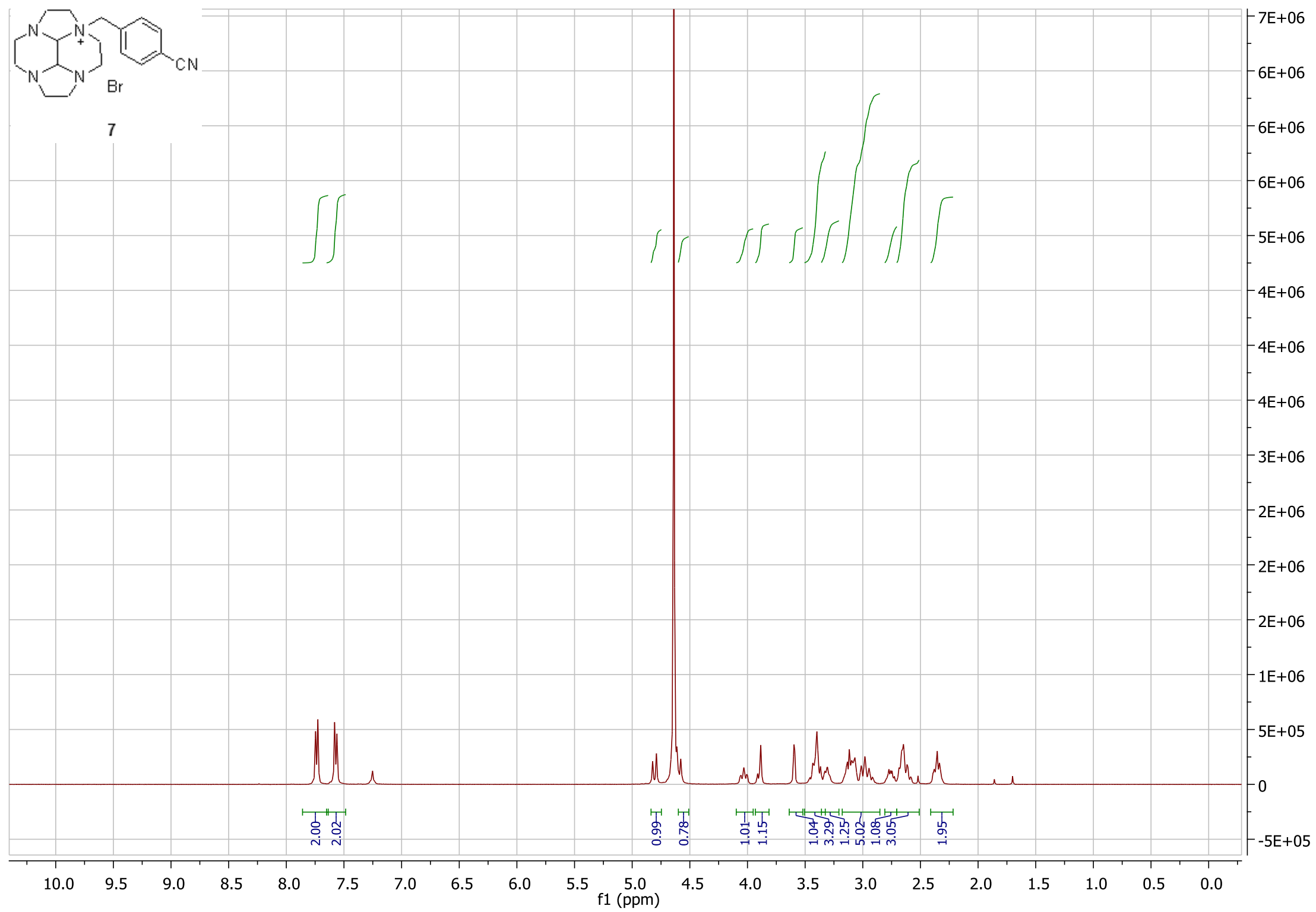




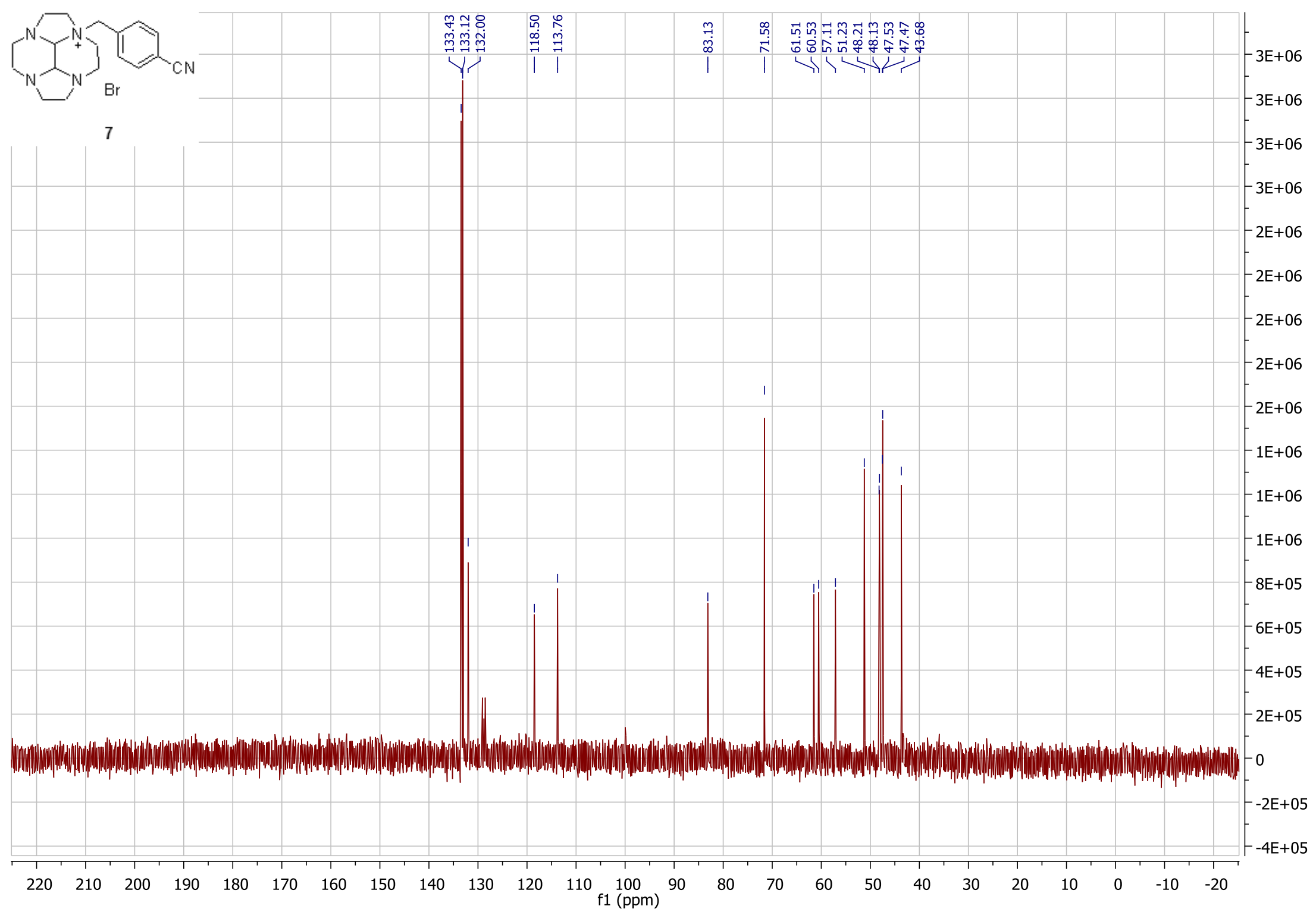




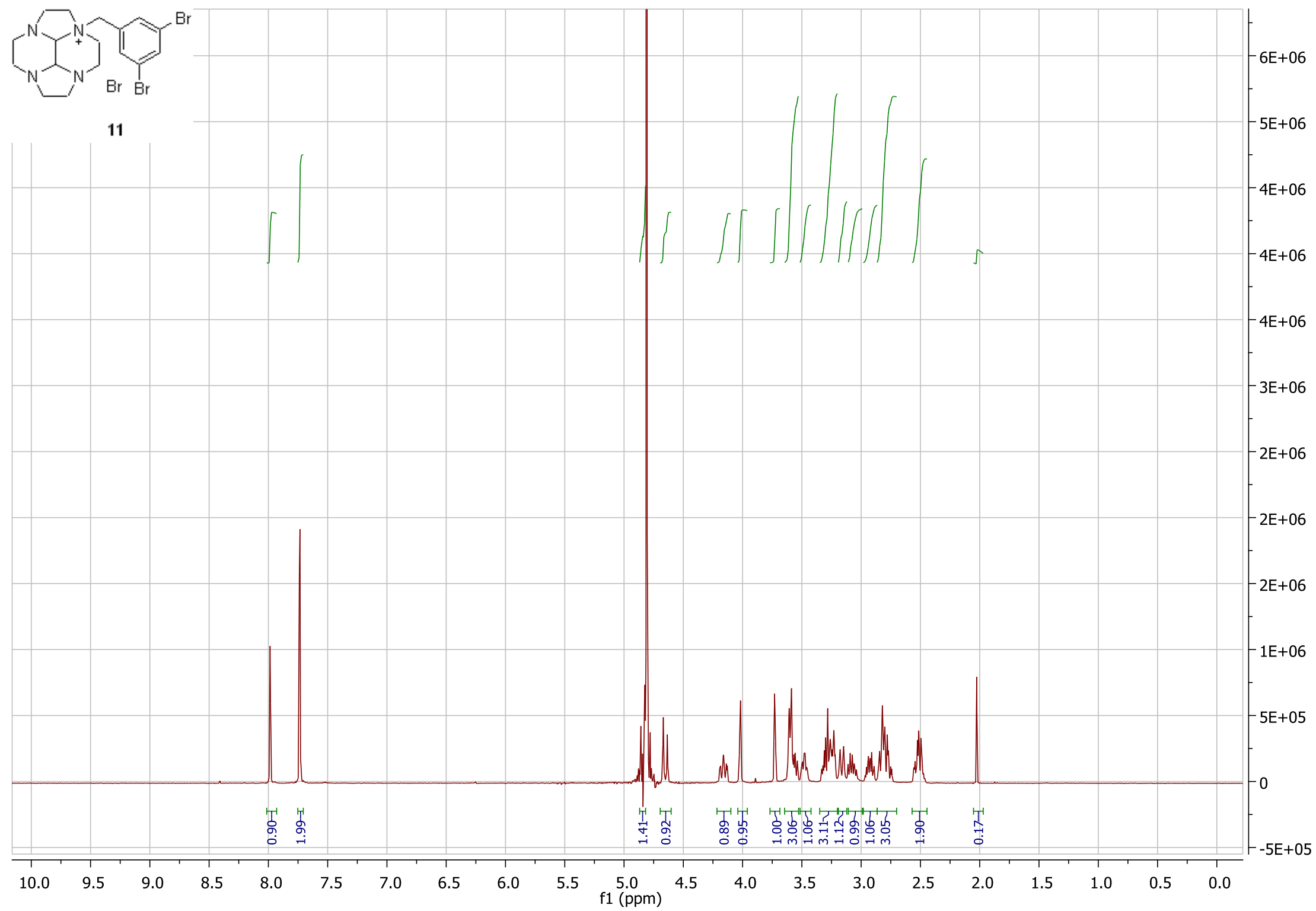




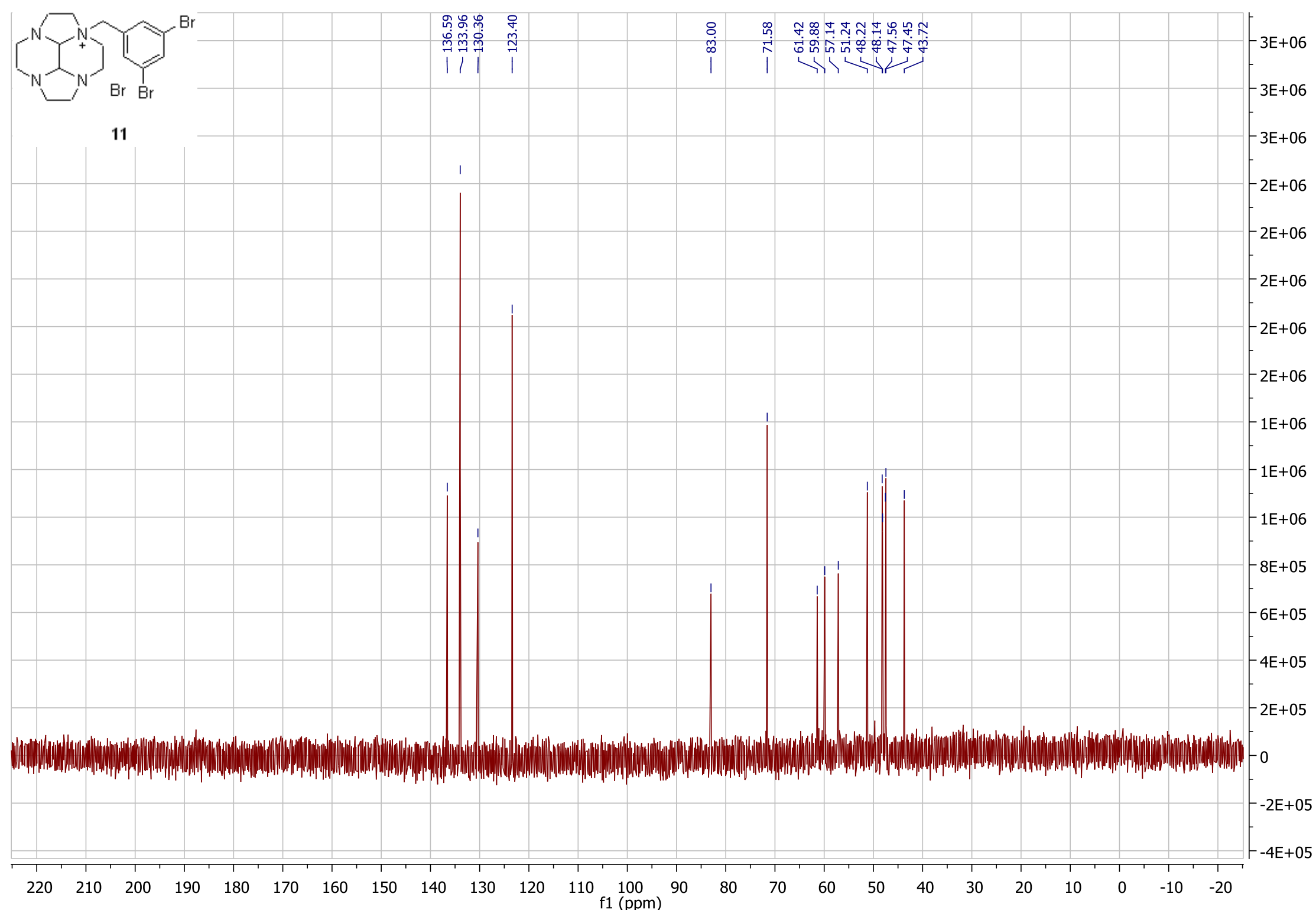




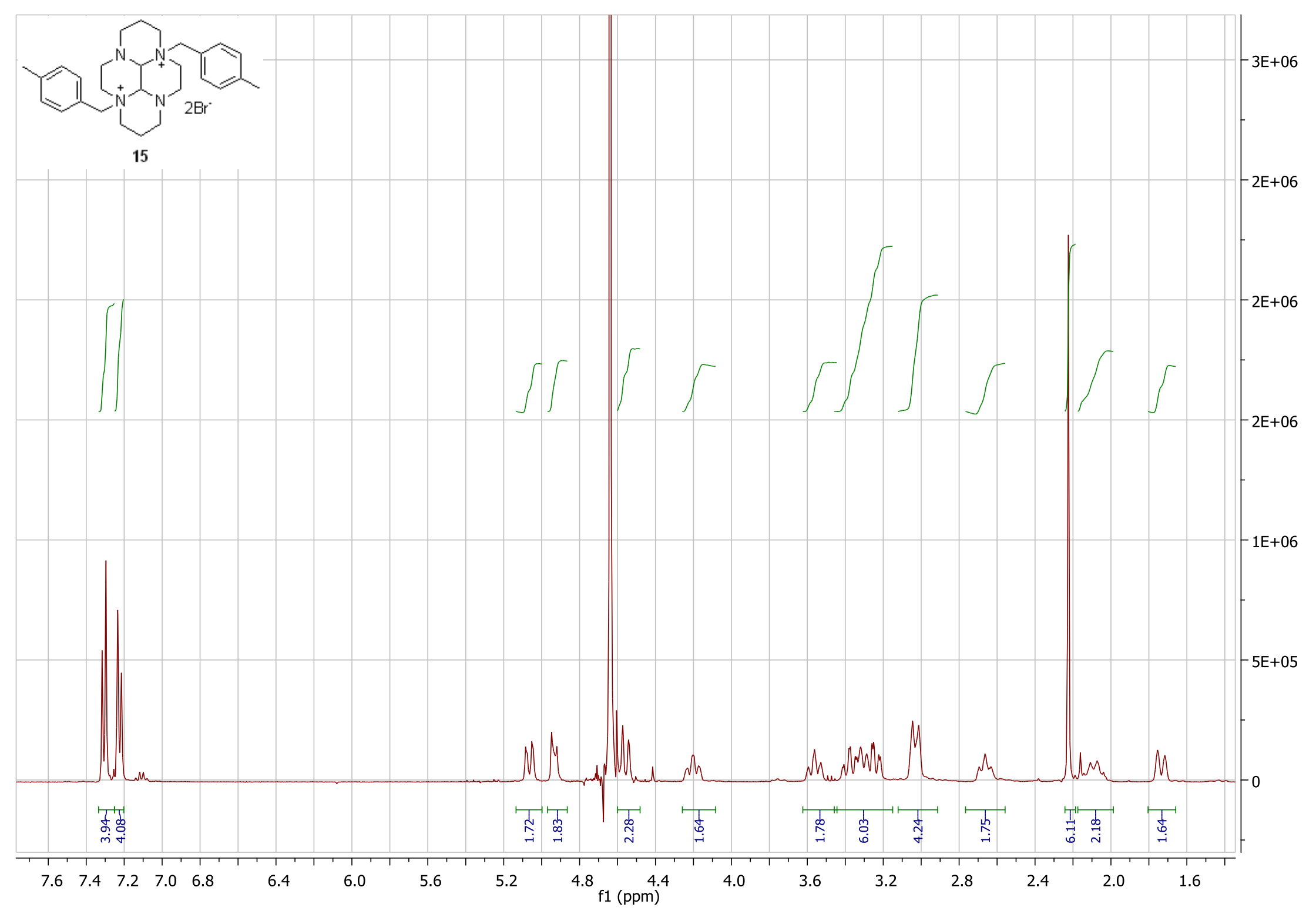




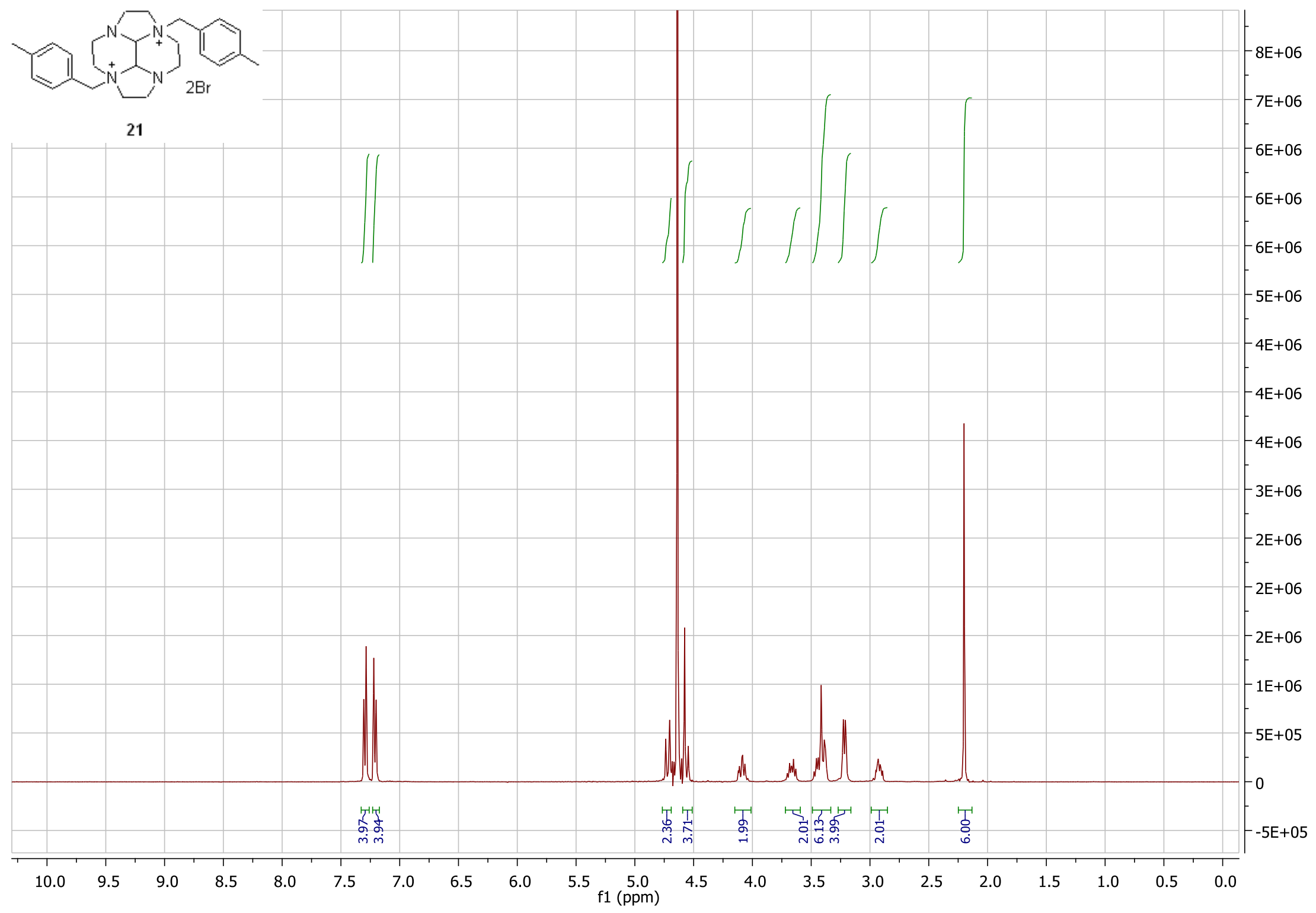




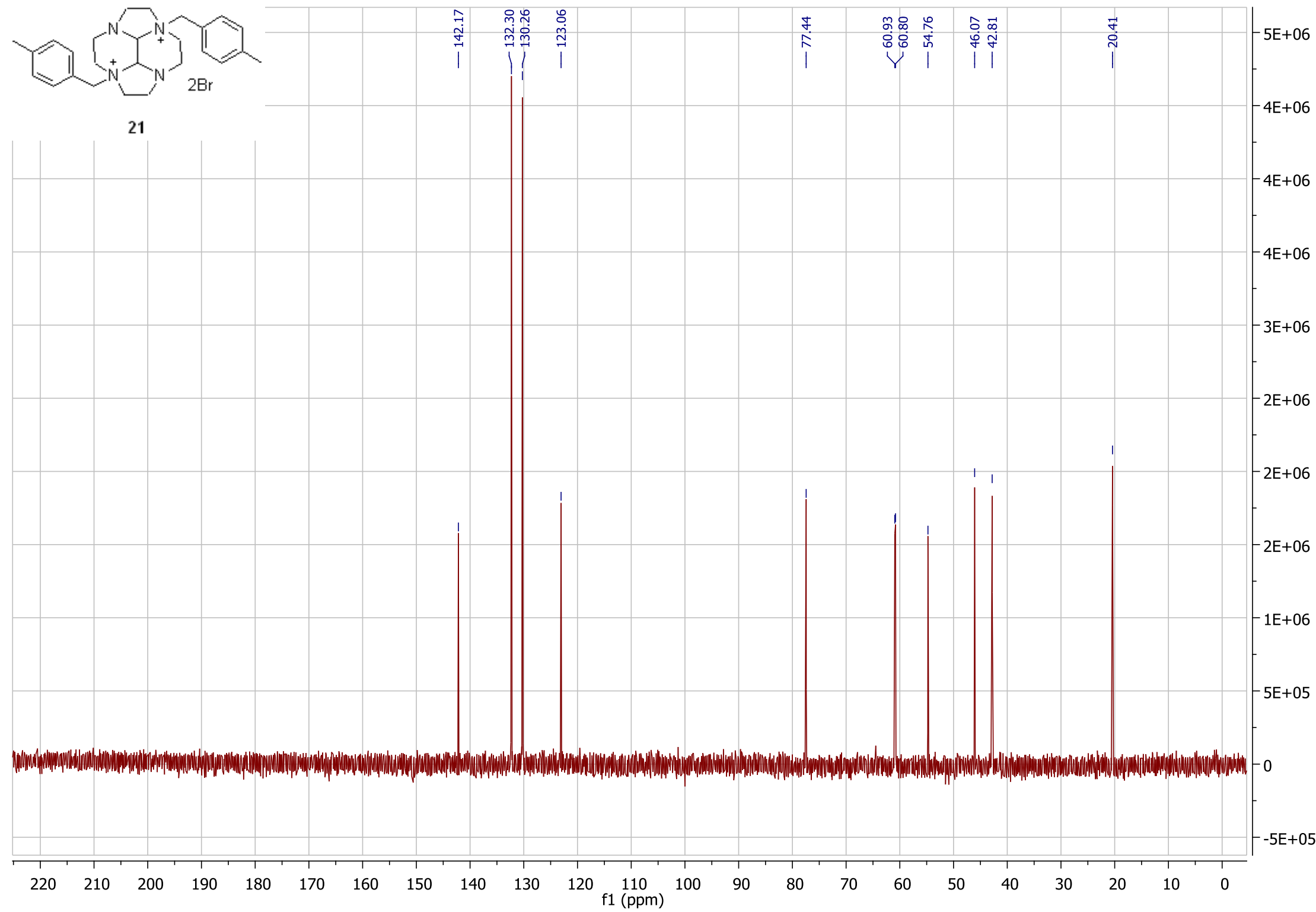




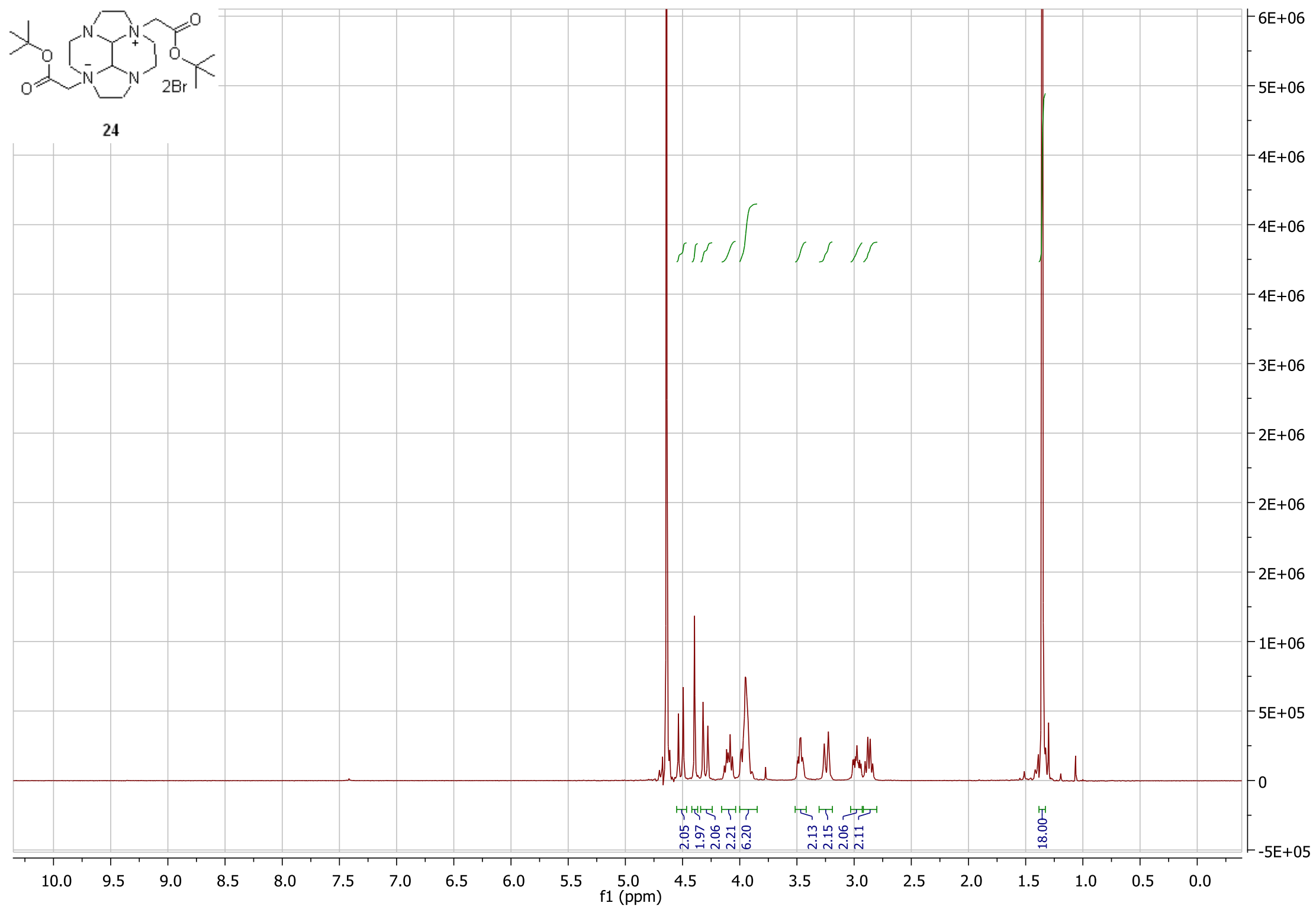




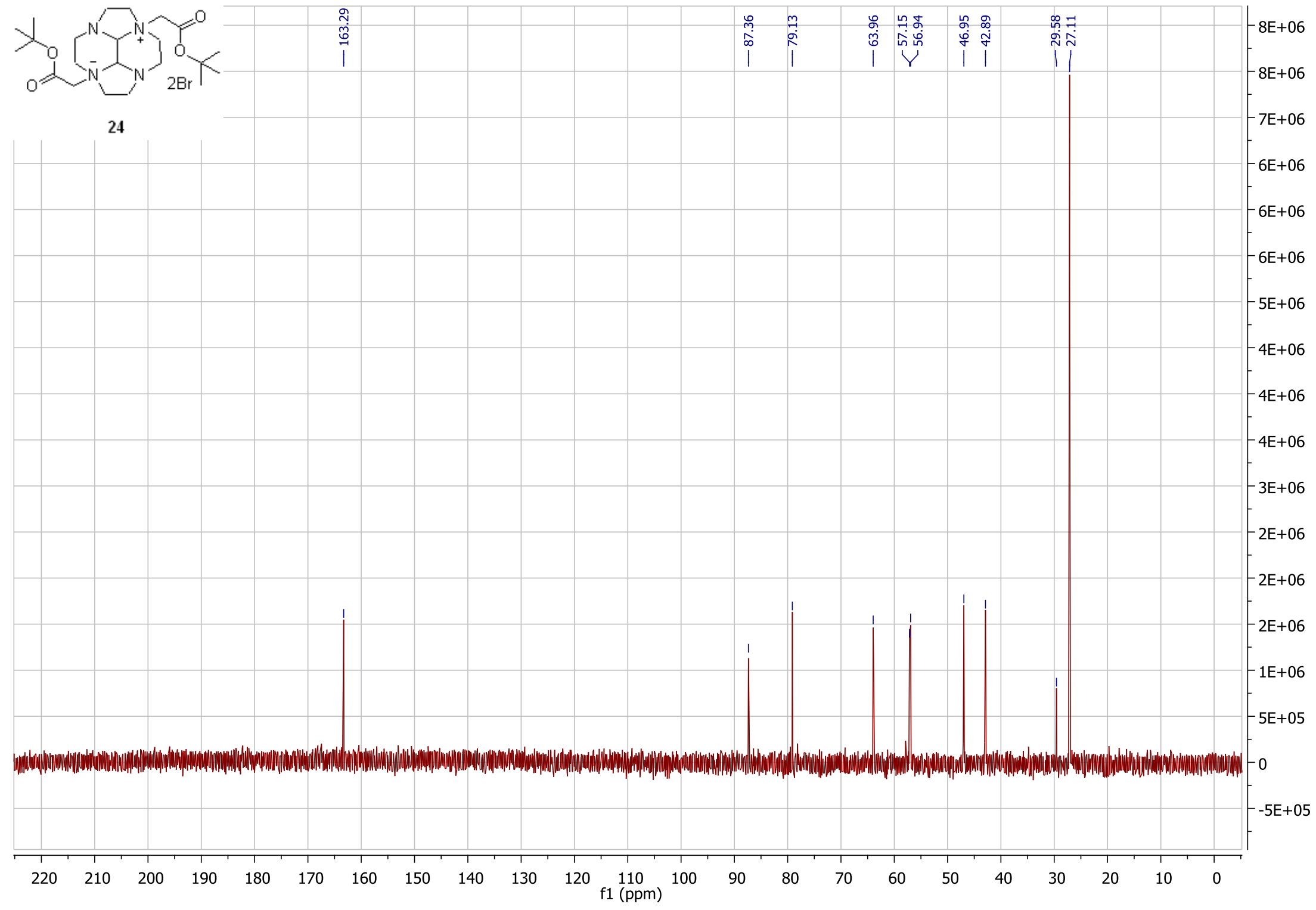




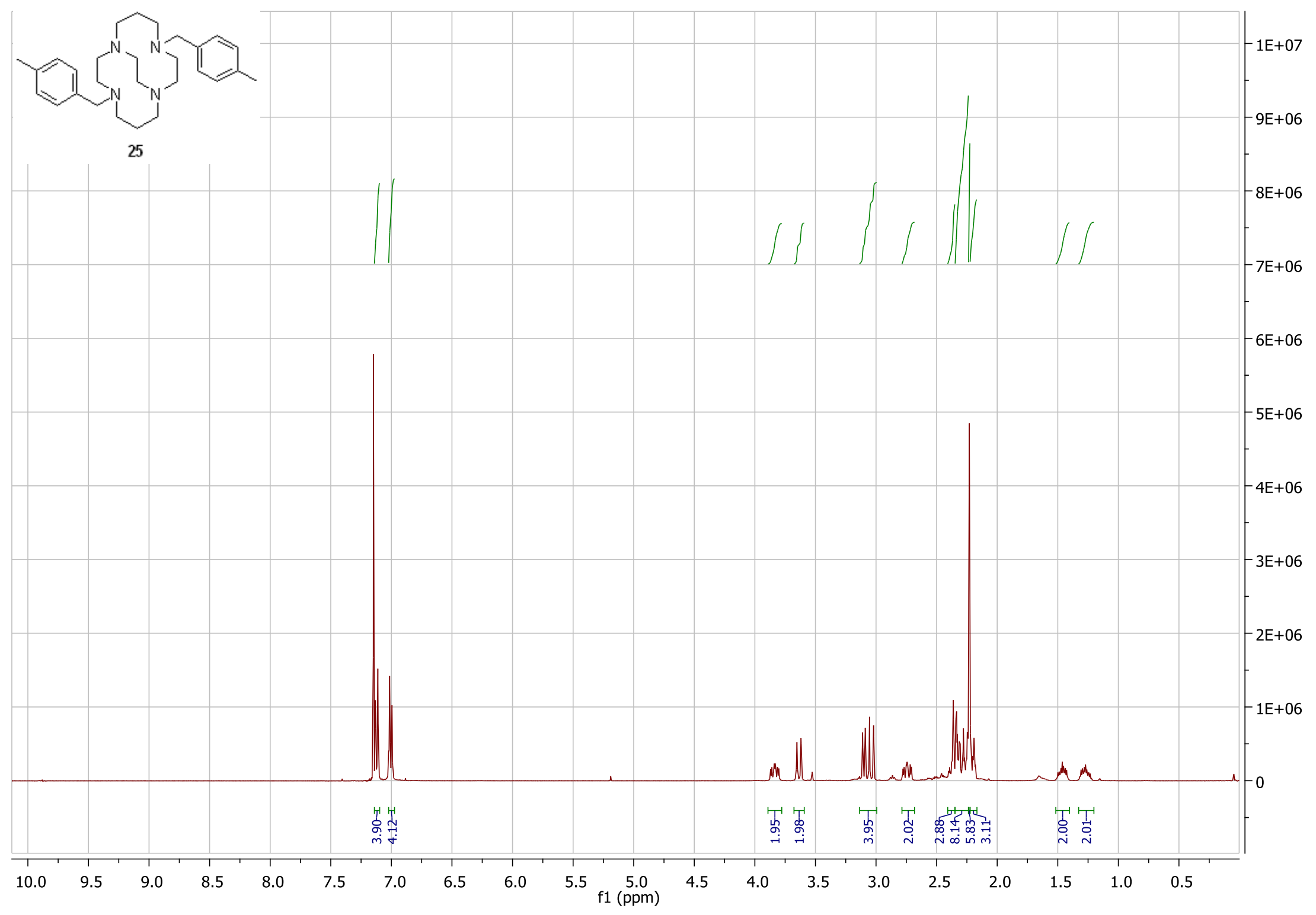




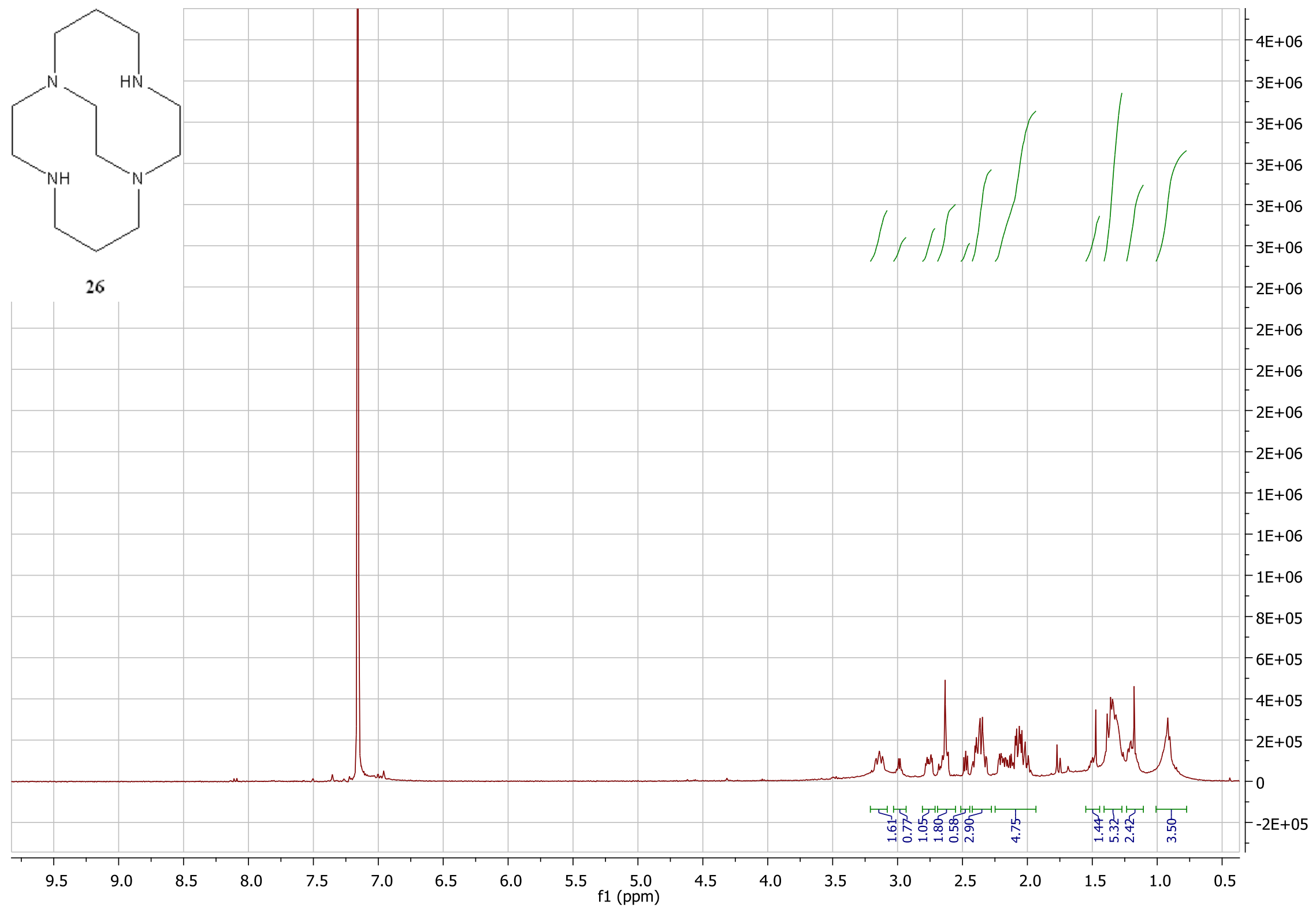




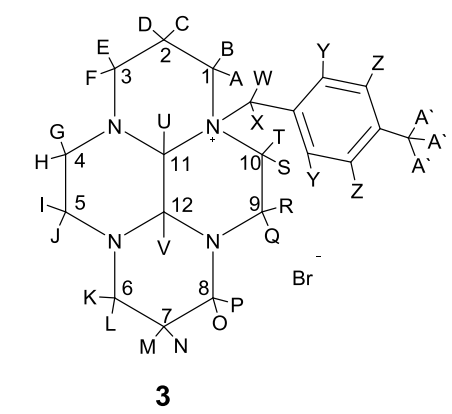

\begin{tabular}{|c|c|c|c|c|}
\hline Atom & ${ }^{1} \mathbf{H}$ & Mult & $\mathbf{J}, \mathbf{H z}$ & COSY \\
\hline $\mathrm{C}$ & 1.46 & $\mathrm{md}$ & 14.2 & $\mathrm{~A}, \mathrm{D}, \mathrm{E}$ or $\mathrm{F}$ \\
\hline $\mathrm{N}$ & 1.77 & md & 14.2 & L,M,P \\
\hline $\mathrm{D}, \mathrm{M}, \mathrm{O}$ & $2.09-2.33$ & $\mathrm{~m}$ & & $\begin{array}{c}\text { A,B,C,E } \\
\text { F,K,L,N,P }\end{array}$ \\
\hline $\mathrm{A}^{\top}$ & 2.39 & $\mathrm{~S}$ & & $\mathrm{Z}$ \\
\hline $\mathrm{H}, \mathrm{J}$ & $2.43-2.51$ & $\mathrm{~m}$ & & $\mathrm{I}, \mathrm{G}$ \\
\hline $\mathrm{A}$ & 2.62 & $\mathrm{td}$ & $3.3 ; 12.5$ & $\mathrm{~B}, \mathrm{C}, \mathrm{D}$ \\
\hline $\begin{array}{c}\text { B,E,F,G, } \\
\text { Q,P,T }\end{array}$ & $2.95-3.15$ & $\mathrm{~m}$ & & $\begin{array}{c}\mathrm{A}, \mathrm{C}, \mathrm{D}, \mathrm{H}, \mathrm{I}, \mathrm{J}, \mathrm{M} \\
\text {,N,O,R,S }\end{array}$ \\
\hline $\mathrm{R}$ & $3.17-3.29$ & $\mathrm{~m}$ & & $\mathrm{Q}, \mathrm{S}, \mathrm{T}$ \\
\hline $\mathrm{K}$ & $3.29-3.36$ & $\mathrm{md}$ & 12.9 & L,M,N \\
\hline $\mathrm{I}, \mathrm{L}$ & $3.44-3.59$ & $\mathrm{~m}$ & & $\mathrm{G}, \mathrm{H}, \mathrm{J}, \mathrm{K}, \mathrm{M}, \mathrm{N}$, \\
\hline $\mathrm{V}$ & 3.65 & $\mathrm{~S}$ & & $\mathrm{U}$ \\
\hline $\mathrm{S}$ & 4.18 & $\mathrm{td}$ & $3.7 ; 13.0$ & $\mathrm{Q}, \mathrm{R}, \mathrm{T}$ \\
\hline $\mathrm{U}$ & 4.35 & $\mathrm{~d}$ & 1.8 & $\mathrm{~V}$ \\
\hline $\mathrm{W}$ & 4.76 & $\mathrm{~d}$ & 13.4 & $\mathrm{X}$ \\
\hline$X$ & 4.99 & $\mathrm{~d}$ & 13.4 & $\mathrm{~W}$ \\
\hline $\mathrm{Y}$ & 7.17 & $\mathrm{~d}$ & 8.4 & $\mathrm{Z}$ \\
\hline$Z$ & 7.21 & $\mathrm{~d}$ & 8.4 & $\mathrm{Y}$ \\
\hline
\end{tabular}

\begin{tabular}{|c|c|}
\hline $\boldsymbol{\delta}, \mathbf{p p m}$ & Atom, ${ }^{\mathbf{1 3}} \mathbf{C}, \mathbf{H M Q C}$ \\
\hline 18.58 & $\mathrm{C}_{\mathrm{CD}}$ or $\mathrm{C}_{\mathrm{NM}}$ \\
\hline 18.96 & $\mathrm{C}_{\mathrm{CD}}$ or $\mathrm{C}_{\mathrm{NM}}$ \\
\hline 20.99 & $\mathrm{C}_{\mathrm{A}}$ \\
\hline 42.56 & $\mathrm{C}_{\mathrm{IJ}}$ \\
\hline 47.20 & $\mathrm{C}_{\mathrm{RQ}}$ \\
\hline 49.18 & $\mathrm{C}_{\mathrm{ST}}$ \\
\hline 51.92 & $\mathrm{C}_{\mathrm{EF}}$ \\
\hline 52.58 & $\mathrm{C}_{\mathrm{OP}}$ \\
\hline 53.88 & $\mathrm{C}_{\mathrm{HG}}$ \\
\hline 54.55 & $\mathrm{C}_{\mathrm{AB}}$ \\
\hline 60.26 & $\mathrm{C}_{\mathrm{KL}}$ \\
\hline 63.08 & $\mathrm{C}_{\mathrm{XW}}$ \\
\hline 70.15 & $\mathrm{C}_{\mathrm{U}}$ \\
\hline 82.24 & $\mathrm{CV}$ \\
\hline 123.12 & $\mathrm{C}_{\mathrm{Y}}-\mathrm{C}_{-} \mathrm{C}_{\mathrm{Y}}$ \\
\hline 130.48 & $\mathrm{C}_{\mathrm{Y}}$ \\
\hline 133.77 & $\mathrm{C}_{\mathrm{Z}}$ \\
\hline 142.49 & $\mathrm{CZ}_{-}-\mathrm{C}_{-}$ \\
\hline
\end{tabular}






22 


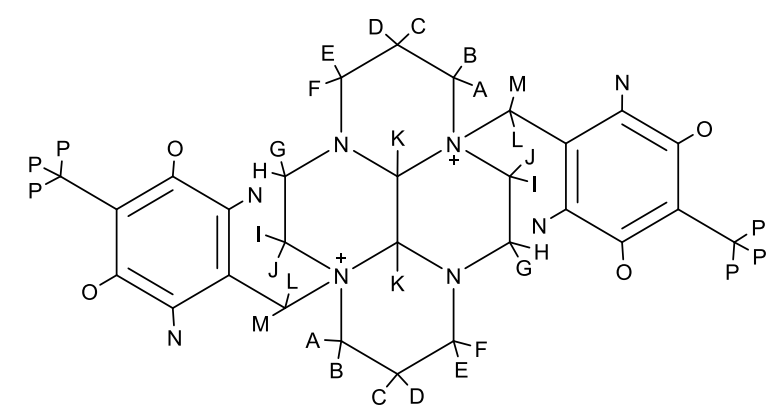

15

\begin{tabular}{|c|c|c|c|c|}
\hline Atom, ${ }^{\mathbf{1}} \mathbf{H}$ & $\boldsymbol{\delta}, \mathbf{p p m}$ & $\mathbf{M u l t}$ & $\mathbf{J}, \mathbf{H z}$ & $\mathbf{F D Q - C O S Y}$ \\
\hline $\mathrm{C}$ & 1.88 & $\mathrm{~d}$ & 15.2 & $\mathrm{~A}, \mathrm{D}, \mathrm{E}$ \\
\hline $\mathrm{D}$ & $2.31-2.17$ & $\mathrm{~m}$ & & $\mathrm{~B}, \mathrm{C}, \mathrm{F}$ \\
\hline $\mathrm{P}$ & 2.37 & $\mathrm{~s}$ & & $\mathrm{O}$ \\
\hline $\mathrm{E}$ & 2.81 & $\mathrm{~d}$ & 12.5 & $\mathrm{C}, \mathrm{D}, \mathrm{F}$ \\
\hline $\mathrm{F}$ & 3.18 & $\mathrm{~d}$ & 12.5 & $\mathrm{E}, \mathrm{D}$ \\
\hline $\mathrm{G}$ & 3.18 & $\mathrm{~d}$ & 12.5 & $\mathrm{H}, \mathrm{I}$ \\
\hline $\mathrm{J}$ & $3.59-3.33$ & $\mathrm{~m}$ & & $\mathrm{G}, \mathrm{H}, \mathrm{I}$ \\
\hline $\mathrm{B}$ & $3.59-3.33$ & $\mathrm{~m}$ & & $\mathrm{~A}, \mathrm{D}$ \\
\hline $\mathrm{H}$ & $3.59-3.33$ & $\mathrm{~m}$ & & $\mathrm{G}, \mathrm{I}, \mathrm{J}$ \\
\hline $\mathrm{A}$ & 3.71 & $\mathrm{t}$ & 12.3 & $\mathrm{~B}, \mathrm{C}$ \\
\hline $\mathrm{I}$ & 4.35 & $\mathrm{t}$ & 11.5 & $\mathrm{G}, \mathrm{H}, \mathrm{J}$ \\
\hline $\mathrm{L}$ & 4.71 & $\mathrm{~d}$ & 13.0 & $\mathrm{M}$ \\
\hline $\mathrm{K}$ & 5.08 & $\mathrm{~d}$ & 8.1 & \\
\hline $\mathrm{M}$ & 5.21 & $\mathrm{~d}$ & 13.0 & $\mathrm{~L}$ \\
\hline $\mathrm{O}$ & 7.37 & $\mathrm{~d}$ & 7.9 & $\mathrm{~N}, \mathrm{P}$ \\
\hline $\mathrm{N}$ & 7.45 & $\mathrm{~d}$ & 7.9 & $\mathrm{~N}$ \\
\hline
\end{tabular}

\begin{tabular}{|l|l|}
\hline $\boldsymbol{\delta}, \mathbf{p p m}$ & Atom, ${ }^{\mathbf{1 3}} \mathbf{C}, \mathbf{H M Q C}$ \\
\hline 18.53 & $\mathrm{C}_{\mathrm{CD}}$ \\
\hline 20.98 & $\mathrm{C}_{\mathrm{p}}$ \\
\hline 46.53 & $\mathrm{C}_{\mathrm{IJ}}$ \\
\hline 47.34 & $\mathrm{C}_{\mathrm{EF}}$ \\
\hline 51.81 & $\mathrm{C}_{\mathrm{AB}}$ \\
\hline 60.91 & $\mathrm{C}_{\mathrm{HG}}$ \\
\hline 62.88 & $\mathrm{C}_{\mathrm{LM}}$ \\
\hline 77.22 & $\mathrm{C}_{\mathrm{K}}$ \\
\hline 122.08 & $\mathrm{C}_{-} \mathrm{C}_{\mathrm{P}}$ \\
\hline 130.60 & $\mathrm{C}_{\mathrm{O}}$ \\
\hline 133.67 & $\mathrm{C}_{-} \mathrm{C}_{\mathrm{LM}}$ \\
\hline 142.88 & $\mathrm{C}_{\mathrm{N}}$ \\
\hline & \multicolumn{2}{|l}{} \\
& \\
& \\
& \\
& \\
&
\end{tabular}


rosion 


\section{Crystallographic data}

\section{Crystallographic data for compound 15}

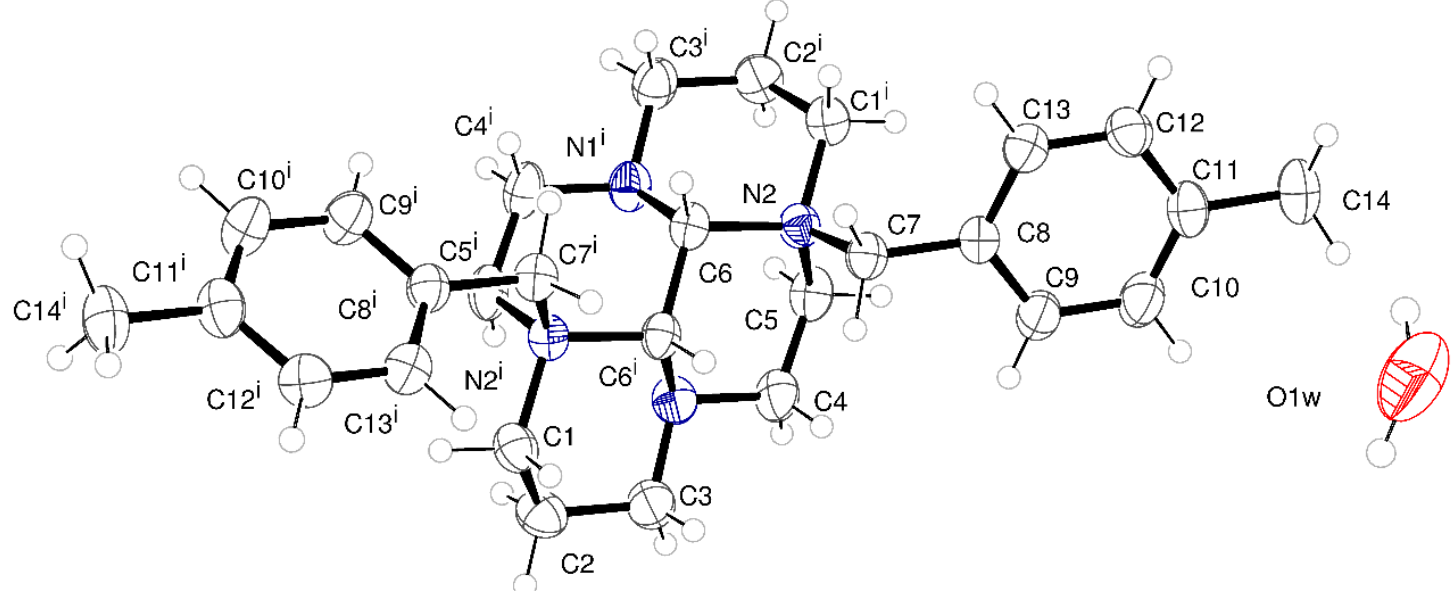

$\mathrm{Br} 1$

Figure 1: ORTEP plot with atoms drawn as $50 \%$ probability ellipsoids. The full macrocycle is represented. Symmetry equivalent atoms are generated by the operator $i=-x, y, 1.5-z$.

The macrocycle resides on a twofold screw axis within the crystal and the second half is generated from the first by application of this symmetry operation. There is limited hydrogen bonding within the structure between the water and the bromide anions. 
Table 1. Crystal data and structure refinement for compound 15.

Identification code

Empirical formula

Structural formula

Formula weight

Temperature

Wavelength

Crystal system

Space group

Unit cell dimensions

Volume

Z

Density (calculated)

Absorption coefficient

$\mathrm{F}(000)$

Crystal size

Theta range for data collection

Index ranges

Reflections collected

Independent reflections

Completeness to theta $=25.242^{\circ}$

Refinement method

Data / restraints / parameters

Goodness-of-fit on $\mathrm{F}^{2}$

Final $\mathrm{R}$ indices [I $>2 \operatorname{sigma}(\mathrm{I})]$

$\mathrm{R}$ indices (all data)

Absolute structure parameter

Largest diff. peak and hole compound 15 (sja16_14)

$\mathrm{C} 28 \mathrm{H} 42 \mathrm{Br} 2 \mathrm{~N} 4 \mathrm{O}$

$\left(\mathrm{C}_{28} \mathrm{H}_{40} \mathrm{~N}_{4}\right)$ 2(Br). $\mathrm{H}_{2} \mathrm{O}$

610.47

$150(2) \mathrm{K}$

$0.71073 \AA$

Orthorhombic

C 2221

$$
\begin{array}{ll}
\mathrm{a}=10.7048(7) \AA & \alpha=90^{\circ} . \\
\mathrm{b}=13.9924(8) \AA & \beta=90^{\circ} . \\
\mathrm{c}=18.8717(13) \AA & \gamma=90^{\circ} .
\end{array}
$$

2826.7(3) $\AA^{3}$

4

$1.434 \mathrm{Mg} / \mathrm{m}^{3}$

$2.895 \mathrm{~mm}^{-1}$

1264

$0.400 \times 0.400 \times 0.100 \mathrm{~mm}^{3}$

2.396 to $29.282^{\circ}$.

$-14 \leq \mathrm{h} \leq 14,-19 \leq \mathrm{k} \leq 19,-25 \leq 1 \leq 25$

10839

$3821[\mathrm{R}(\mathrm{int})=0.1380]$

$99.9 \%$

Full-matrix least-squares on $\mathrm{F}^{2}$

$3821 / 2 / 164$

0.914

$\mathrm{R} 1=0.0626, \mathrm{wR} 2=0.1441$

$\mathrm{R} 1=0.0842, w \mathrm{R} 2=0.1532$

$0.20(2)$

1.827 and -0.914 e. $\AA^{-3}$ 


\section{Crystallographic data for Compound 21 (Form I)}

Compound 21 was found to crystallise in two different forms that differed in the level of solvation. Form I was produced by diffusion of methanol into an ether solution of 21. During the analysis of form I, some of the solvent present (ether/methanol) evaporated and a second solvate, Form II, was obtained. Each form contains the same macrocycle.

Form I has composition $\left(\mathrm{C}_{26} \mathrm{H}_{36} \mathrm{~N}_{4}\right)$. 2(Br). $\mathrm{H}_{2} \mathrm{O}$

Form II has composition $\left(\mathrm{C}_{26} \mathrm{H}_{36} \mathrm{~N}_{4}\right) 2(\mathrm{Br}) . \mathrm{CH}_{3} \mathrm{OH} .1 .5 \mathrm{H}_{2} \mathrm{O}$

21 (Form 1) displays beautiful crystallographic disorder of the macrocycle. Within the solid state there is disorder in the orientation of the cylen core. Standard techniques were used to model this disorder (see CIF file). The structure and disorder are exemplified in the ORTEP plots below.

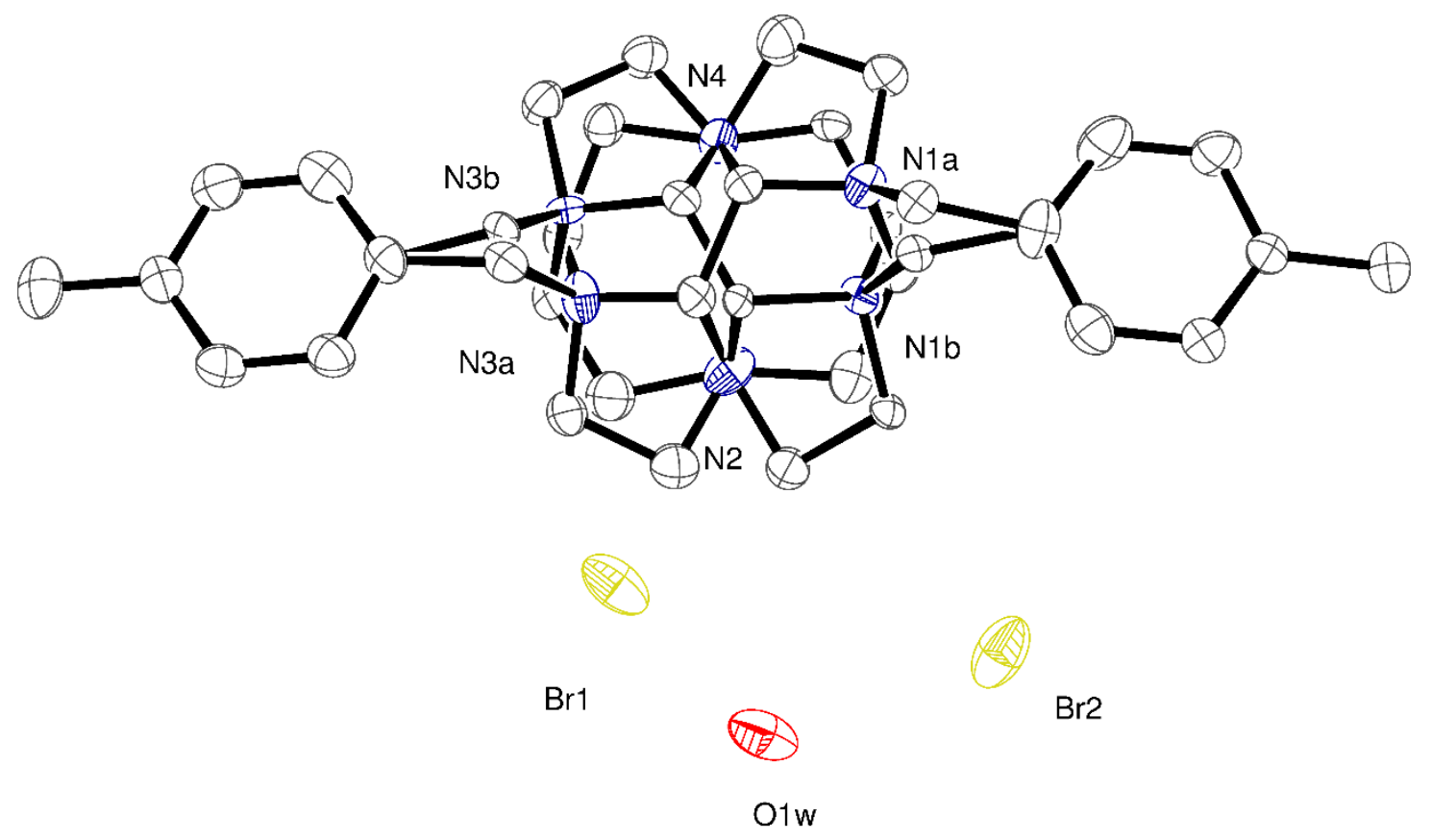

Figure 2: ORTEP plot of 21 (Form I) with atoms drawn as $\mathbf{5 0 \%}$ probability ellipsoids. The full macrocycle is represented. For clarity only selected atoms are labelled and hydrogen atoms are omitted.

The molecule present is much clearer if the two different orientations of the cyclen are coloured differently as shown below. The major orientation (60.1(7)\%) of the ring is coloured black and the minor orientation $(39.9(7) \%)$ is coloured red.

A diagram to confirm the correct product is shown in Figure 4. 


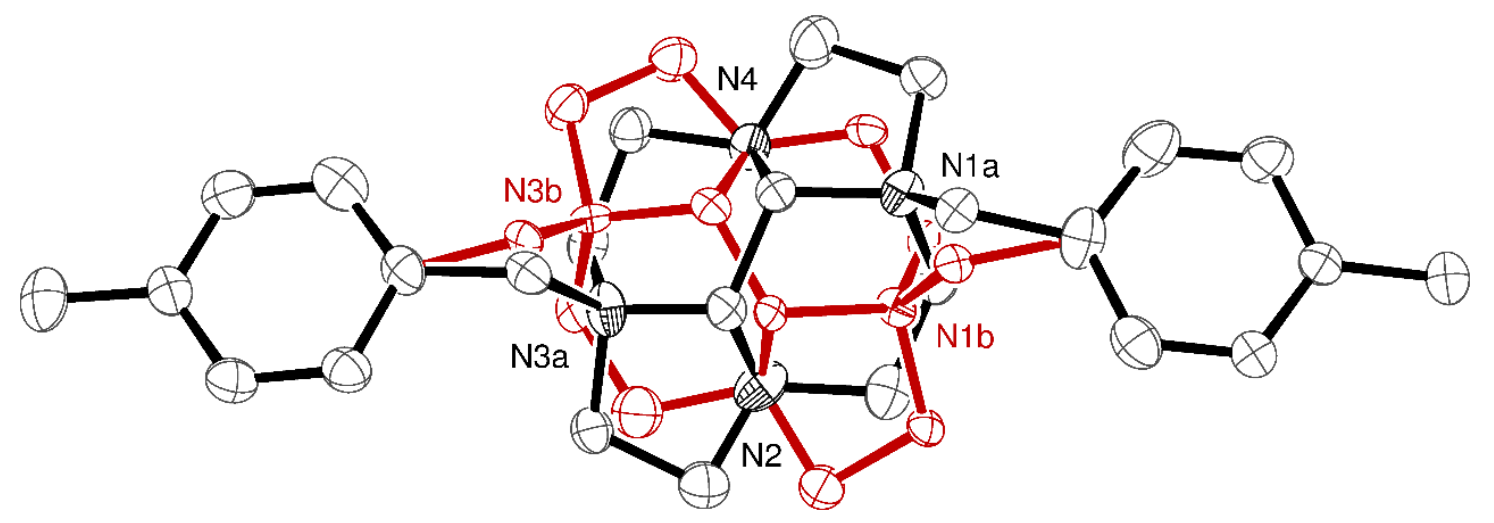

$\mathrm{Br} 1$

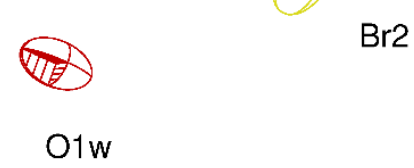

Figure 3: ORTEP plot of Form I with atoms drawn as 50\% probability ellipsoids. The two different orientations of the full macrocycle are coloured differently. For clarity only selected atoms are labelled and hydrogen atoms are omitted. The atoms labelled $\mathrm{N} 2$ and $\mathrm{N} 4$ are common to both orientations.
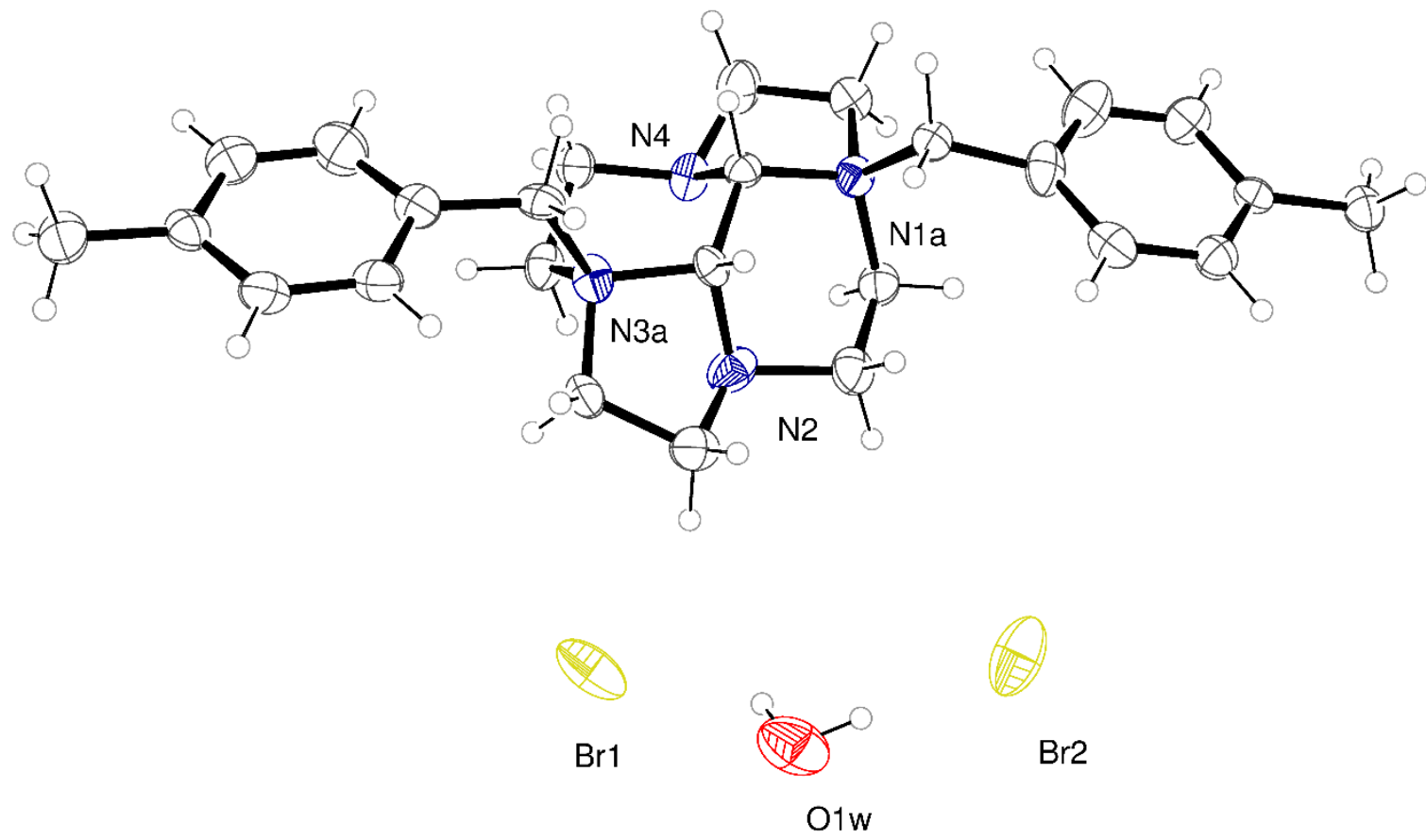

Figure 4: ORTEP plot with atoms of major component of Form I drawn as 50\% probability ellipsoids. 
Table 2. Crystal data and structure refinement for compound 21 (Form I).

Identification code

Empirical formula

Structural formula

Formula weight

Temperature

Wavelength

Crystal system

Space group

Unit cell dimensions

Volume

$\mathrm{Z}$

Density (calculated)

Absorption coefficient

$\mathrm{F}(000)$

Crystal size

Theta range for data collection

Index ranges

Reflections collected

Independent reflections

Completeness to theta $=25.242^{\circ}$

Absorption correction

Max. and min. transmission

Refinement method

Data / restraints / parameters

Goodness-of-fit on $\mathrm{F}^{2}$

Final $\mathrm{R}$ indices [I $>2 \operatorname{sigma}(\mathrm{I})]$

$\mathrm{R}$ indices (all data)

Largest diff. peak and hole

\section{compound 21 (Form I)}

C26 H38 Br2 N4 O

$\left(\mathrm{C}_{26} \mathrm{H}_{36} \mathrm{~N}_{4}\right)$ 2(Br). $\mathrm{H}_{2} \mathrm{O}$

582.42

150(2) K

$0.71073 \AA$

Monoclinic

P $2{ }_{1} / \mathrm{n}$

$\mathrm{a}=14.5400(9) \AA$

$\alpha=90^{\circ}$.

$\mathrm{b}=10.1743(8) \AA$

$\beta=102.778(5)^{\circ}$.

$\mathrm{c}=18.1427(11) \AA$ $\gamma=90^{\circ}$.

2617.5(3) $\AA^{3}$

4

$1.478 \mathrm{Mg} / \mathrm{m}^{3}$

$3.123 \mathrm{~mm}^{-1}$

1200

$0.260 \times 0.260 \times 0.220 \mathrm{~mm}^{3}$

1.630 to $26.149^{\circ}$.

$-18 \leq \mathrm{h} \leq 18,-11 \leq \mathrm{k} \leq 12,-22 \leq 1 \leq 22$

13153

$5127[\mathrm{R}(\mathrm{int})=0.0711]$

$98.1 \%$

Analytical

0.5509 and 0.4948

Full-matrix least-squares on $\mathrm{F}^{2}$

5127 / 274 / 435

1.050

$\mathrm{R} 1=0.0998, \mathrm{wR} 2=0.2221$

$\mathrm{R} 1=0.1519, \mathrm{wR} 2=0.2426$

1.284 and -1.458 e. $\AA^{-3}$ 


\section{Crystallographic data for Compound 21 (Form II)}

The second crystal form of compound 21 does not contain disorder. Its composition differs from Form I only in the solvent present.

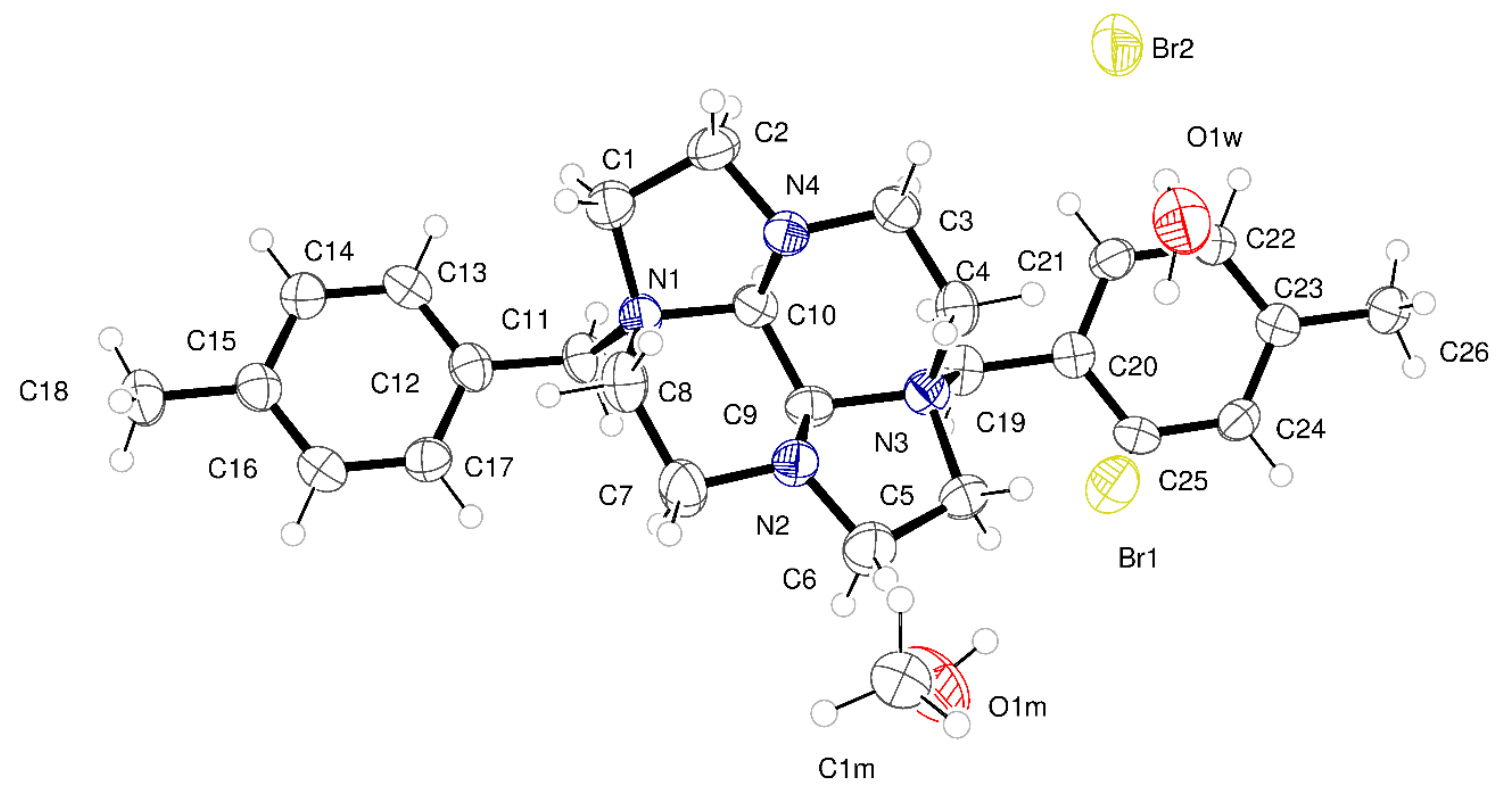

Figure 5: ORTEP plot for Form II with atoms drawn as 50\% probability ellipsoids. The full macrocycle is represented. For clarity only selected atoms are labelled and hydrogen atoms are omitted. 
Table 3. Crystal data and structure refinement for compound 21 (Form I).

Identification code

Empirical formula

Structural formula

Formula weight

Temperature

Wavelength

Crystal system

Space group

Unit cell dimensions

Volume

$\mathrm{Z}$

Density (calculated)

Absorption coefficient

$\mathrm{F}(000)$

Crystal size

Theta range for data collection

Index ranges

Reflections collected

Independent reflections

Completeness to theta $=25.242^{\circ}$

Refinement method

Data / restraints / parameters

Goodness-of-fit on $\mathrm{F}^{2}$

Final R indices [I $>2 \operatorname{sigma}(\mathrm{I})]$

$\mathrm{R}$ indices (all data)

Largest diff. peak and hole

\section{compound 21 (Form II)}

C27 H43 Br2 N4 O2.50

$\left(\mathrm{C}_{26} \mathrm{H}_{36} \mathrm{~N}_{4}\right)$ 2(Br). $\mathrm{CH}_{3} \mathrm{OH}$. $1.5 \mathrm{H}_{2} \mathrm{O}$

623.47

150(2) K

$0.71073 \AA$

Monoclinic

P $2{ }_{1} / \mathrm{n}$

$\mathrm{a}=9.7180(5) \AA$

$\alpha=90^{\circ}$.

$\mathrm{b}=22.6793(13) \AA$

$\beta=102.826(4)^{\circ}$.

$\mathrm{c}=13.0099(7) \AA$ $\gamma=90^{\circ}$

2795.8(3) $\AA^{3}$

4

$1.481 \mathrm{Mg} / \mathrm{m}^{3}$

$2.933 \mathrm{~mm}^{-1}$

1292

$0.420 \times 0.160 \times 0.120 \mathrm{~mm}^{3}$

1.796 to $25.641^{\circ}$.

$-11 \leq \mathrm{h} \leq 8,-24 \leq \mathrm{k} \leq 27,-15 \leq 1 \leq 15$

14043

$5246[\mathrm{R}($ int $)=0.0771]$

$99.7 \%$

Full-matrix least-squares on $\mathrm{F}^{2}$

$5246 / 8 / 336$

1.013

$\mathrm{R} 1=0.0534, \mathrm{wR} 2=0.1430$

$\mathrm{R} 1=0.0819, \mathrm{wR} 2=0.1558$

1.139 and -1.819 e. $\AA^{-3}$ 